\title{
Enumeration of CD34+ haemopoietic stem cells: comparative study of the performance of the SYSMEX XN-1000 hematology analyzer in a dual-platform approach versus a single-platform approach
}

\author{
A. Berhili ${ }^{1,2}$ (1) S. Lamrabet ${ }^{1} \cdot$ M. Bensalah ${ }^{1}$ - O. Bouayadi ${ }^{1} \cdot$ A. Elyagoubi ${ }^{1} \cdot$ E. Sebbar $^{1} \cdot$ O. Nassiri $^{1} \cdot$ B. Mouhoub ${ }^{1}$. \\ I. Elmezgueldi ${ }^{1} \cdot$ R. Seddik ${ }^{1,2}$
}

Received: 2 September 2020 / Accepted: 1 December 2020 / Published online: 8 January 2021

(C) The Author(s), under exclusive licence to Springer-Verlag GmbH, DE part of Springer Nature 2021

\begin{abstract}
Data regarding the quantification of CD34+ hematopoietic stem cell HSC in dual-platform (DP) approach using a hematological analyzer featuring dedicated nRBC channel are limited if not inexistent. The objective of this study is to compare the performance of the DP approach on quantification of CD34+ HSC using a new generation of hematological analyzer featuring dedicated nRBC channel, Sysmex XN-1000, with the single-plate (SP) form approach using Beckman Coulter's Navios flow cytometer. Using the International Society for Hematotherapy and Transplantation protocol, a total of 86 samples were analyzed in multiple myeloma patients planned for autologous HSC transplantation. The Beckman Coulter's Stem-Kit ${ }^{\mathrm{TM}}$ reagents were used to perform both approaches DP and SP. The count of CD34+ cells of the SP deduced directly from the software that manage the Navios flow cytometry was compared to CD34+ cell count obtained by DP. The DP approach was made by combining flow cytometry analysis and the Sysmex XN-1000 hematological cell analyzer data. The analysis of the agreement of the results between these two approaches using the Bland-Altman diagram gave a bias of one cell/ $\mu$ l. As for the equation of the right of Passing-Bablok, it is $Y(\mathrm{SP})=0.166667+0.969697 X(\mathrm{DP})$. The slight differences in CD34+ stem cell counting between these two approaches did not achieve statistical significance. Both SP and DP approaches were effective and yield similar results. DP seems to be a simple and affordable approach that is well suited to the needs of laboratories where this type of analyzer is available.
\end{abstract}

Keywords Mobilized peripheral blood stem cells $\cdot$ CD34+ $\cdot$ Flow cytometry $\cdot$ Sysmex XN-1000

\section{Introduction}

Since its clinical introduction in 1986, the transplantation of mobilized hematopoietic stem cells into peripheral blood (PBSC) has become the most common transplantation procedure performed in medicine [1]. It is widely used to treat blood disorders, malignant tumors, and genetic abnormalities.

A. Berhili

a.berhili@ump.ac.ma

1 Hematology Laboratory, Mohammed VI University Hospital, BP 4806 Oujda Universite, 60049 Oujda, Morocco

2 Genetics Unit, Medical Sciences Research Laboratory, Faculty of Medicine and Pharmacy, Mohammed Premier University, Oujda, Morocco
To collect a satisfactory graft, the choice of the best timing to initiate the cytapheresis cycle is a critical factor, and this process depends essentially on the number of CD34+ stem cells mobilized in the peripheral blood [2]. Flow cytometry analysis is currently one of the fastest and most accurate solutions to vigilantly monitor the effectiveness of the entire CD34+ hematopoietic stem cell (HSC) mobilization process.

Several approaches have been proposed to enumerate CD34+ HSCs by flow cytometry. Among these, two are the most commonly used: The first method is called a single platform (SP), which is considered a reference method where $\mathrm{CD} 34+\mathrm{HSCs}$ are marked in the presence of a known number of beads (fluorospheres) in a tube. The cytometer relates the number of beads counted to the number of CD34+ cell counted, and the results are expressed in percentage and absolute numbers of the CD34+ HSCs by applying a precise formula $[3,4]$. The second method called dual platform (DP) combines two instruments, flow cytometry and a hematological 
analyzer. Here, the absolute number of CD34+ HSCs is deduced from the percentage of CD34+ cells evaluated via flow cytometry and the absolute number of nucleated cells and/or the number of white blood cells (WBCs) obtained by a hematological analyzer [4].

Published works comparing these two approaches known as single and dual platforms as regards of enumeration of CD34+ HSCs are limited, and even less those comparing these two approaches through the application of the ISHAGE protocol. In several published works, the DP approach was carried out using hematological analyzer cells whose operating principles are basically working on the impedance principle (coulter principle). With such analyzers, there is a potential risk of a false increase of the total number of white blood cells due to the integration of nucleated red blood cell (nRBC) levels into white blood cell levels. Leading to a higher risk of error when calculating CD34+ HSCs by DP technique [5-7].

The aim of this study is to evaluate the contributions of the nRBC channel technology in a Sysmex XN-1000 hematological analyzer in the enumeration of CD34+ HSCs by the DP approach and to compare these results to the SP, reference method recommended by ISHAGE.

\section{Materials and methods}

\section{Sample collection}

In order to choose the timing for harvesting mobilized stem cells in multiple myeloma patients programmed for autologous stem cell transplantation, mobilized peripheral blood hematopoietic stem cell (HSPC) samples were obtained in EDTA tubes and cytapheresis bags under the approval of the Research Ethics Board. Primary analysis of the received samples was performed using the Sysmex XN-1000 hematological analyzer which is maintained calibrated and regularly controlled using 3-level quality controls (Hematology Control $\mathrm{XN}$ Check ${ }^{\mathrm{TM}}$ ). The WBC count of each sample was adjusted to $15,000 \mathrm{WBC} / \mu \mathrm{l}$ by dilution with a phosphate-buffered serum (PBS). All samples were processed simultaneously by both SP and DP methods within $2 \mathrm{~h}$ of sampling.

\section{Sample preparation}

Using Beckman Coulter's Stem-Kit Reagents (Beckman Coulter Life Sciences, Indianapolis, USA), three tubes were prepared for the analysis of each peripheral blood tube (46 samples) and each cytapheresis tube (40 samples). The first 2 tubes labeled "tube 1" and "tube 2 " were prepared identically by staining $100 \mu \mathrm{l}$ of the sample initially deposited by reverse pipetting with $20 \mu \mathrm{l}$ of CD 45-FITC clone J33 and CD34-PE clone 581 and $20 \mu \mathrm{l}$ of viability marker 7-AAD viability dye. The third tube labeled "control" was prepared identically to tubes "1" and " 2 "; the only difference is that the sample is stained with isoclonic marker (CD 45-FITC clone $\mathrm{j} 33$ /IsoClonic Controle-PE clone 581) instead of CD 45-FITC clone j33/CD34-PE clone 581. The three tubes 1, 2, and control were gently vortexed for $5 \mathrm{~s}$ and incubated under obscurity for $20 \mathrm{~min}$ at room temperature. Erythrocyte lysis was performed by adding $2 \mathrm{ml}$ of the lysis solution ( $\mathrm{NH} 4 \mathrm{Cl}$ lysing solution) diluted to $1 / 10$ th by distilled water into each of the three tubes. The whole unit was vortexed for $5 \mathrm{~s}$ then incubated at $18-25{ }^{\circ} \mathrm{C}$ for 10 min under obscurity. A total of $100 \mu \mathrm{l}$ of previously homogenized stem-count fluorosphere was added using a calibrated pipette into each of the three tubes, then one last vortex passage to all tubes for $5 \mathrm{~s}$. A positive control was prepared using Stem-Trol control cells (Beckman Coulter Life Sciences, Indianapolis, USA), according to the manufacturer's recommendations and treated under the same conditions as the patients' samples. The acquisition was made immediately on the Beckman Coulter Navios flow cytometer. The control is validated if the obtained Stem-Trol count is within $\pm 15 \%$ of the Stem-Trol control cell concentration stated on the vial label.

\section{Calculations}

Enumeration of viable CD34+ HSCs by these two approaches (SP and DP) was carried out by applying the recommendations of the ISHAGE (International Society of Hematotherapy and Graft Engineering) protocol [9] designated for the enumeration of CD34+ HSCs by flow cytometry.

The results of the CD34+ HSC count by SP technique were derived directly from the software that manages the Navios cytometer version 1.3. A multiplication by the dilution factors is required if the sample was initially diluted with PBS. Since the SP technique is very sensitive to pipetting errors, WBC count and lymphocyte count obtained by cytometry were compared with those obtained with the Sysmex XN-1000 hematological analyzer. They can be used as an internal control on each sample, and have allowed us, in addition to a positive control, to ensure the correct preparation of dilutions and the acquisition of our stem count.

The results of the CD34+ HSC count by DP technique were obtained by combining the percentage of CD34+ HSCs in the analyzed sample deduced from the flow cytometer and the absolute WBC count obtained by the XN1000 hematological analyzer. The absolute number of CD34+ HSCs was the result of a WBC calculation *\% CD34+ HSCs.

\section{Statistical analysis}

A study of the concordance between these two methods (SP and DP) was performed using the 2016 EXCEL software and MedCalc version 14.8.1.0 by calculating the correlation concordance coefficients of LIN $(\rho c)[10]$ and Pearson $(\rho)$ and 
analyzing the Bland and Altman graph [11] to quantify the agreement between the two techniques. A regression study was conducted using the Passing-Bablok model [12], which does not require specific assumptions about sample distribution and measurement errors.

\section{Results}

The results of the CD34+ HSC counting on 86 samples collected from patients with multiple myeloma utilizing the DP and SP techniques are included in Table 1. The WBC count of all samples was adjusted around $15.10^{3} \mathrm{~GB} / \mu \mathrm{l}$ before being analyzed by both SP and DP techniques. The median of WBC count in our series is $15.01510^{3} \mathrm{WBC} / \mu \mathrm{l}$. The means and medians obtained by these approaches (SP and DP) are almost identical for stem cells mobilized in peripheral blood (PB) and cytapheresis samples. The comparison of means of the CD34+ HSC counts measured by both methods reveals a difference of $0.2174 \mathrm{CD} 34+$ stem cells $/ \mu$ l stem cells; $95 \%$ CI 9428 to 3.3776; $P=0.8916$ for $\mathrm{PB}$ and a difference of 1.8250 CD34+ stem cells $/ \mu 1,95 \%$ CI -4.4977 to 8.1477 ; and $P=$ 0.5672 for PBSC. These results show that the differences between means of CD34+ HSCs measured by the SP and DP method in PB and PBSC are not statistically significant. On the other hand, the nRBC count by the Sysmex XN-1000 hematological analyzer for each sample type shows the presence of a significant number of nucleated red blood cells. The medians are $20 \mathrm{nRBC} / \mu \mathrm{l}$ and $40 \mathrm{nRBC} / \mu$ l for peripheral blood and cytapheresis samples respectively for the same amount of WBC (15.0.15 $10^{3} \mathrm{~GB} / \mu \mathrm{l}$ in our series). They represent between 0.2 and $0.3 \%$ depending on the sampling nature, and the range is $80 \mathrm{nRBC} / \mu \mathrm{l}$ for both types of samples (Table 2).

Table 1 CD34+ mobilized stem cell count results
Table 2 Nucleated red blood cell (nRBC) count results by Sysmex XN1000 hematological analyzer

\begin{tabular}{lllll}
\hline Sample & Average & Median & Range & \% of WBC count \\
\hline PB & 31.1 & 20 & 80 & 0.2 \\
PBSC & 37 & 40 & 80 & 0.3 \\
\hline
\end{tabular}

The statistical analysis of the CD34+ HSC numeration results shows a very good correlation between these two techniques (Table 3). Lin's concordance correlation coefficient $(\rho c)=0.9864$, Pearson's $\rho$ (precision) $=0.9924$, and a bias correction factor $\mathrm{Cb}$ (precision) $=0.9939$, indicating an excellent agreement between two sets of measurements according to the interpretation of Partik et al. [13]. The analysis of the Bland and Altman graph (Fig. 1) reveals that the bias is 1 cell, indicating that the DP method overestimates the number of CD34+ HSCs by an average of one cell compared to the method (SP) which is the reference method. The BlandAltman plotted as percentage of differences (Fig. 2) demonstrates that the bias is $-2.1 \%$ and that the four points to the right of the Bland-Altman plot (Fig. 1) are well within a standard deviation of the overall distribution of differences. The equation of the Passing-Bablok line (Table 4 and Fig. 3) is $Y$ $(\mathrm{SP})=0.166667+0.969697 X(\mathrm{DP})($ the $95 \%$ confidence interval of this slope is 0.9130 to 1.0000 ). The Cusum test did not reveal a significant deviation from linearity $(P=0.78)$.

\section{Discussion}

Peripheral blood stem cell transplant has progressively become the most common transplant procedure performed in medicine, replacing bone marrow transplantation as a source

\begin{tabular}{llllll}
\hline Sample & PB & & & PBSC \\
\cline { 2 - 3 } Methods & DP_1_CD_34_1 & SP_1_CD_34_1 & & DP_1_CD_34_1 & SP_1_CD_34_1 \\
\hline Sample size & 46 & 46 & 40 & 40 \\
Median & 10 & 10 & 29.5 & 28.5 \\
Arithmetic mean & 11.63 & 11.42 & 32.5000 & 30.6750 \\
95\% CI for the mean & 9.343 to 13.917 & 9.169 to 13.656 & 27.690 to 37.309 & 26.416 to 34.933 \\
Standard deviation & 7.7010 & 7.55 & 15.0384 & 13.3155 \\
Range & 36 & 35 & 62 & 53 \\
$T$ test* (assuming equal variances) & 0.2174 & & & \\
Difference & $P=0.8916$ & & 1.8250 & \\
Two-tailed probability & $P=0.5672$ & \\
\hline
\end{tabular}

$P B$ peripheral blood, $P B S C$ mobilized peripheral blood stem cells (in cytapheresis samples)

*Significance level $(\alpha)=5 \%$

${ }^{1}$ Dual platform: obtained by combining flow cytometry and the $\mathrm{XN}-1000$ hematological analyzer

${ }^{2}$ Single platform: obtained by flow cytometry (Beckman Coulter's Navios) 
Table 3 Concordance correlation results of CD34+ mobilized hematopoietic stem cell counts performed by both SP and DP approaches

\begin{tabular}{ll}
\hline Sample size & 86 \\
\hline Concordance correlation coefficient & 0.9864 \\
$95 \%$ confidence interval & 0.9806 to 0.9904 \\
Pearson $\rho$ (precision) & 0.9924 \\
Bias correction factor $C_{\mathrm{b}}$ (accuracy) & .9939 \\
\hline
\end{tabular}

of stem cells in nearly $100 \%$ of autologous patients and approximately $75 \%$ of allogeneic transplant patients [1]. The success of this procedure is mainly based on the number of transfused CD34+ HSCs. Following the integration of flow cytometry as a promising tool for CD34+ HSC counting in 1980, several methods and protocols have since been described to provide a reliable and reproducible number of CD34+ HSCs. Currently, the ISHAGE protocol is the most widely used protocol [14]. The quantification of CD34+ HSCs according to the ISHAGE protocol by flow cytometry could be performed using two approaches, the so-called SP and DP approaches. These two approaches have both benefits and drawbacks. The SP technique is precise but fairly rigorous to be maintained in routine due to the specific properties of the beads. An abnormal bead count can be encountered with this method, in particular during processing of protein-free or lowprotein cell samples. This abnormality can occur as a direct consequence of sedimentation or sticking of the beads against the walls of the polystyrene tubes in various situations: During vortexes, electrostatic charges caused by the friction of the polystyrene tubes with the rubber housing of the vortex tube lead to a noticeable change in the concentration of the beads in the tube causing a false CD34+ HSC enumeration. This observed phenomenon can also occur spontaneously over the

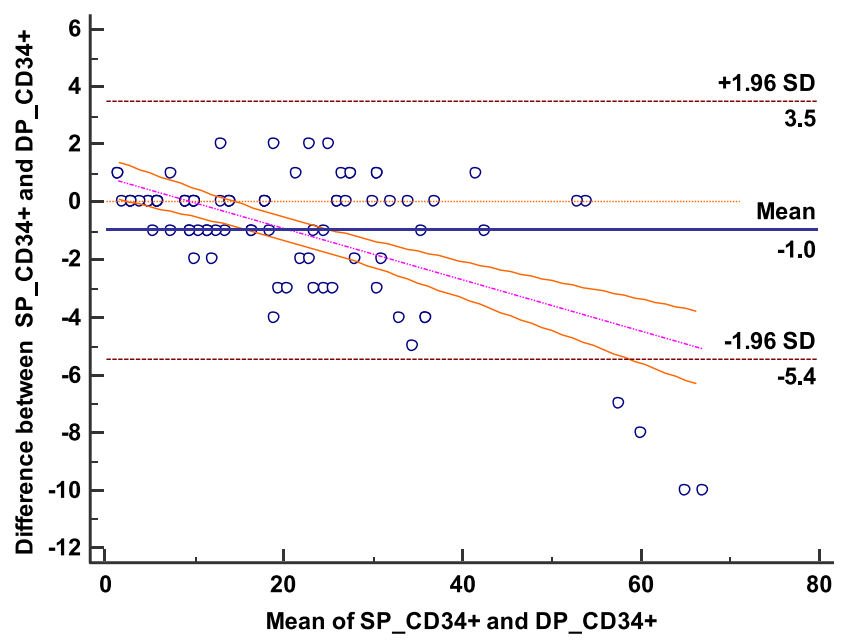

Fig. 1 Bland-Altman diagram of differences comparing CD34+ hematopoietic stem cell count results obtained with both SP and DP approaches

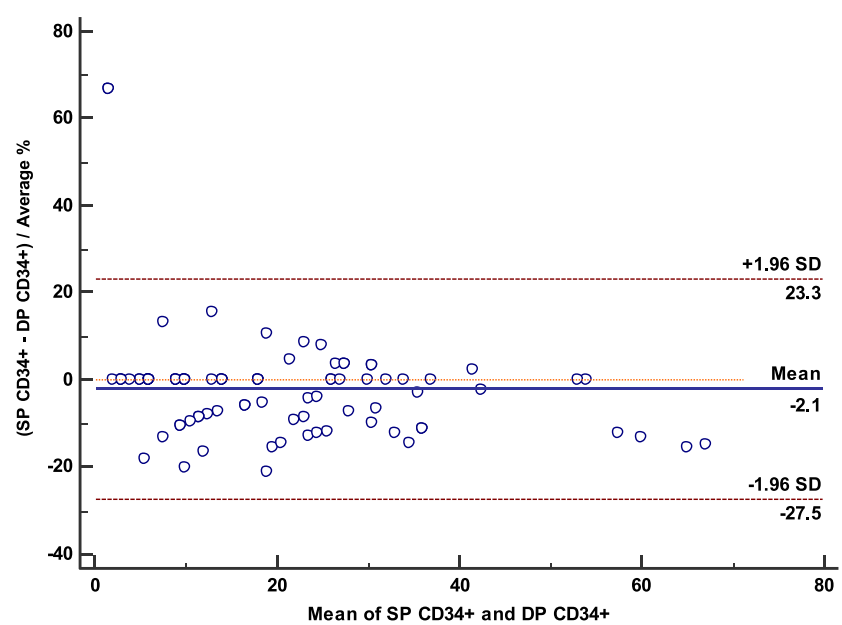

Fig. 2 Bland-Altman plot of percent difference comparing CD34+ hematopoietic stem cell count results obtained with both SP and DP approaches

time and called "vanishing counting bead phenomenon" [15]. Furthermore, this method necessitates an average of two counts to provide a final report of CD34+ HSC count. If the variation in values exceeds $10 \%$ between two counts, it becomes imperative to repeat the test, which increases the required time and cost to perform the $\mathrm{CD} 34+$ cell count using this approach [16].

The DP technique presents a critical problem when processing samples rich in nRBCs. The latter was found in nearly all onco-hematological diseases. And they have also been frequently encountered in mobilized peripheral blood hematopoietic stem cells [20]. In this case, we are experiencing a false evaluation of CD34+ HSCs due to the integration of nRBC levels in the total number of WBC $[18,19]$. This increase is in the order of $1 \%$ for the treatment of mobilized peripheral blood, $3.08 \%$ for the treatment of cytapheresis products and $6.09 \%$ for the treatment of umbilical cord blood [20].

Published works comparing these two approaches known as SP and DP for CD34+ HSC count are limited, and even fewer are those comparing these two approaches through the application of the ISHAGE protocol. According to D Barnett et al. inquiry, SP approach is the best in terms of reducing the inter-laboratory variability [21]. This is due to the determining problem that presents the DP approach of reporting the percentage of CD34+ HSCs in relation to the proportion of leukocytes or total nucleated cells including nRBC [4].

Through this, it is clear that the performance of the DP approach is related to the characteristics of the hematological analyzer, i.e., whether it is designed to give the exact WBC count in the case of analyzing erythroblast-rich samples or these results require microscope review to correct the WBC count. In which case, the DP technique would suffer from the same common weaknesses known to be present in all manual 
Table 4 Results of the regression analysis

\author{
$Y=0.166667+0.969697 X$ \\ Systematic differences \\ Intercept A \\ 0.1667 \\ 95\% CI \\ 0.0000 to 0.5870 \\ Proportional differences \\ Slope B \\ 0.9697 \\ 95\% CI \\ 0.9130 to 1.0000 \\ Random differences \\ Residual standard deviation (RSD) \\ 1.4947 \\ \pm 1.96 RSD interval \\ -2.9296 to 2.9296 \\ Linear model validity \\ Cusum test for linearity \\ No significant deviation from linearity $(P=0.78)$
}

assays, such as intra- and inter-observer reproducibility and sampling errors [22].

Most of the published studies in this regard were based on hematological analyzers that operate on the Coulter principle to perform the DP technique, as Sysmex KX-21 for Ngoma A.M et al. [23], a Coulter counter STKS model for PiedrasRoss J et al. [24], and Sysmex k-1000, Technicon H3 for Gratama JW et al. [4]. All these hematological analyzers, which operation is based on the impedance principle, were reported to have limitations against the presence of nRBC. In addition, most of these studies are limited to correlation analysis and regression analysis to compare these two approaches, although these two parameters do not provide sufficient resolution. According to Keeney $\mathrm{M}$ et al., the use of Bland and Altman's presentation is strongly recommended for the analysis of such a situation [3].

The hematology analyzers used to perform the DP approach in our study are Sysmex XN-1000. This analyzer contains a specific channel (WNR) to differentiate between WBC, basophils, and erythroblasts [25]. Based on the manufacturer's

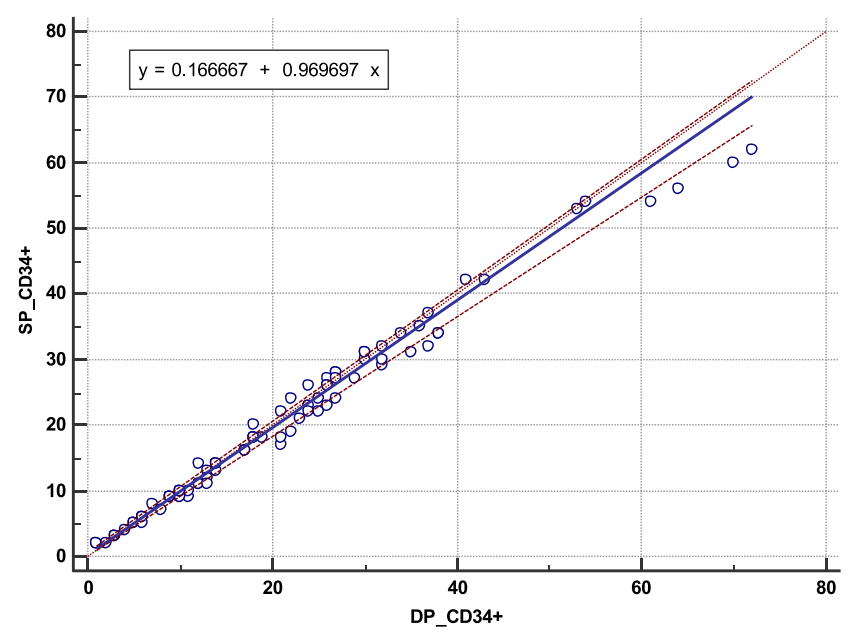

Fig. 3 Correlation diagram according to Passing and Bablok specs, Sysmex XN-1000 model is able to detect and correct automatically the blood count when nRBCs are detected in the analyzed sample with a sensitivity up to $1 \mathrm{nRBC} / 1000 \mathrm{WBC}$ [8].

Statistical analysis of CD34+ HSC count results, by both SP approach using Beckman Coulter's Navios cytometer and DP coupled with the XN-1000 automat, shows a Lin's concordance correlation coefficient ( $\rho c)$ : 0.9864, Pearson's $\rho$ $($ precision $)=0.9924$ and a bias correction factor $\mathrm{Cb}$ (precision $)=0.9939$. These results reflect an excellent agreement between the two sets of measure. The part of the imprecision in our series is $(1-0.9924) /((1-0.9924)+(1-$ $0.9939))=55.47 \%$ and the part of the inaccuracy is $(1-$ $0.9939) /((1-0.9939)+(1-0.9924))=44.53 \%$. The difference observed between the two approaches in our series is due to a lack of precision (55.47\%) more than to a lack of accuracy $(44.53 \%)$. To improve further the consistency between these two approaches, random errors between the two sets of measurement must be reduced.

To confirm the efficiency of this specific channel (WNR) on Sysmex XN-1000 in the CD34+ HSC enumeration by DP approach, we can calculate the accuracy without "this correction" by adding absolute nRBC counts which are detected on Sysmex XN-1000 to the WBC counts initially obtained, then recalculate the CD34+ HSCs according to the DP's guidelines. By doing so, the accuracy without this correction is $\mathrm{Cb}=0.9892$ (compared to $\mathrm{Cb}=0.9939$ found previously with "corrected" WBC count). Based on these results, we can conclude that the accuracy always is better with "corrected" WBC count.

On the other hand, the bias value is one cell, and this means that the "DP" method overestimates the number of CD34+ HSCs on average by one cell/ $\mu$ l compared to the SP method. This difference can be explained by the fact that the hematological analyzer used in DP is basically designed to process whole blood samples drawn in EDTA and to a lesser degree cytapheresis samples drawn in citrate solutions. This 
hypothesis is supported by comparing the differences between means (Table 1) of CD34+ HSC count measured by SP and DP for each type of samples separately $(0.2$ cells for peripheral blood samples versus a bias of 1.8 for cytapheresis samples). The Passing-Bablok regression did not indicate a significant difference between these two approaches. The obtained results are similar to those reported from Chapple P.H. et al. using an Abbott Cell dyn 4000 hematological analyzer that integrates cytometry technology in DP. Chapple P.H found a bias of 1.16 and a linear regression $R^{2}=0.9325$ [26]. These results show that the integration of cytometric technology alongside impedance in medium/high-range hematological analyzers makes this latter in DP approach much more reliable as SP for the quantification of CD34+ HSC count.

Umbilical cord blood is known to be one of the richest sources of HSCs with the highest erythroblast concentrations [17], making them as the most vulnerable element of the DP. In comparison of the umbilical cord blood erythroblast count generated by Sysmex XN-1000 with the gold method which is microscopic count, Kim $\mathrm{H}$ et al. demonstrated the presence of a strong correlation between these two techniques $(r=0.865)$ [25], which makes us expect that DP paired with Sysmex XN-1000 hematological analyzer remains an extremely promising approach in term of processing HSC sources assumed to contain high levels of erythroblasts. However, a verification of the performance of DP coupled with Sysmex XN-1000 on CD34+ HSC counting on umbilical cord blood remains highly recommended. Also, a prospective and multicenter study is encouraged to complete this study.

\section{Conclusion}

The results of the CD34+ HSC count using the DP approach that combines cytometry with the Sysmex XN-1000 hematological analyzer were significantly correlated with the SP approach recommended by ISHAGE. DP seems to be a simple, affordable, and well-adapted approach that suits the needs of laboratories where this type of analyzer is available either as a dedicated method to quantify CD34+ HSCs or as a monitoring procedure to assess the correct execution of the SP technique.

Acknowledgments We gratefully thank Pr ZEGMOUT Mohammed and Ms. TAHRI Maroua for their assistance, Mr. DEHMANI Abdelmalek for the statistical support, and Pr BOUALI Abderrahim for the corrections made to the manuscript.

\section{Compliance with ethical standards}

Conflict of interest The authors declare that they have no conflict of interest.

\section{References}

1. Körbling M, Freireich EJ (2011) Twenty-five years of peripheral blood stem cell transplantation. Blood 117:6411-6416

2. Rosskopf K, Ragg SJ, Worel N, Grommé M, Preijers FW, Braakman E et al (2011) Quality controls of cryopreserved haematopoietic progenitor cells (peripheral blood, cord blood, bone marrow). Vox Sang 101:255-275

3. Keeney M, Chin-yee I, Weir K, Popma J, Nayar R, Sutherland DR (1998) Single platform flow cytometric absolute CD34+ cell counts based on the ISHAGE guidelines. Cytometry 34:61-70

4. Gratama JW, Braakman E, Kraan J, Lankheet P, Levering WH, Van Den Beemd MW et al (1999) Comparison of single and dual-platform assay formats for CD34+ haematopoietic progenitor cell enumeration. Clin Lab Haematol 21:337-346

5. W H B M Levering. External quality assessment in flow cytometry: educational aspects and trends toward improvement. Editorial: Erasmus University Rotterdam -10-24, 2007.

6. Barnett D et al (1999) Guideline for the flow cytometric enumeration of CD34+ haematopoietic stem cells. Prepared by the CD34+ Haematopoietic Stem Cell Working Party. General Haematology Task Force of the British Committee for Standards in Haematology. Clin Lab Haematol 21(5):301-308

7. Gratama JW et al (1998) Flow Cytometric enumeration of CD34+ hematopoietic stem and progenitor cells. Cytometry 34(3):128-142

8. Steffens M (2015) Product fact sheet, added values XN-CBC and XN-DIFF. Sysmex Europe GmbH 1-3

9. Sutherland DR, Anderson L, Keeney M, Nayar R, Chin-Yee I (1996) The ISHAGE guidelines for CD34+ cell determination by flow cytometry. J Hematother 5:213-226

10. Lin L (1989) A concordance correlation coefficient to evaluate reproducibility. Biometrics 45:255-268

11. Bland JM, Altman DG (1986) Statistical methods for assessing agreement between two methods of clinical measurement. Lancet 327:307-310

12. Passing H, Bablok W, Bender R, Schneider B (1988) A general regression procedure for method transformation. Application of Linear Regression Procedures for Method Comparison Studies in Clinical Chemistry, Part III. Journal of Clinical Chemistry and Clinical Biochemistry. Zeitschrift Fur Klinische Chemie Und Klinische Biochemie 26(11):783-790

13. Partik BL, Stadler A, Schamp S, Koller A, Voracek M, Heinz G et al (2002) 3D versus 2D ultrasound: accuracy of volume measurement in human cadaver kidneys. Investig Radiol 37:489-495

14. Whitby A, Whitby L, Fletcher M, Reilly JT, Sutherland DR, Keeney M, Barnett D (2012) ISHAGE protocol: are we doing it correctly? Cytometry B Clin Cytom 82:9-17

15. Brando B, Göhde JW, Scarpati B, D’Avanzo G (2001) The "vanishing counting bead" phenomenon: effect on absolute CD34+ cell counting in phosphate-buffered saline-diluted leukapheresis samples. Cytometry 43:154-160

16. Rahul N, Nitin D, Gaurav D (2017) Single versus dual platform analysis for hematopoietic stem cell enumeration using ISHAGE protocol. Indian J Hematol Blood Transfus 33:370-374

17. Danise P, Maconi M, Barrella F, Di Palma A, Avino D, Rovetti A, Gioia M, Amendola G (2012) Evaluation of nucleated red blood cells in the peripheral blood of hematological diseases. Clin Chem Lab Med (CCLM) 50(2):357-360

18. Hübl W, Iturraspe J, Martinez GA, Hutcheson CE, Roberts CG, Fisk DD et al (1998) Measurement of absolute concentration and viability of CD34+ cells in cord blood and cord blood products using fluorescent beads and cyanine nucleic acid dyes. Cytometry $34: 121-127$ 
19. Geneviève F, Godon A, Marteau-Tessier A, Zandecki M (2012) Automated hematology analysers and spurious counts Part 2. Leukocyte count and differential. Ann Biol Clin 70:141-154

20. Fornas O, Domingo JC, Marin P, Petriz J (2002) Flow cytometricbased isolation of nucleated erythroid cells during maturation: an approach to cell surface antigen studies. Cytometry 50:305-312

21. Barnett D, Granger V, Kraan J, Whitby L, Reilly JT, Papa S, Gratama JW, for and on behalf of the CD34 Task Force of the European Working Group of Clinical Cell Analysis (EWGCCA) (2000) Reduction of intra- and interlaboratory variation in CD34+ stem cell enumeration using stable test material, standard protocols and targeted training. Br J Haematol 108:784-792

22. Hwang D, Dorfman D, Hwang D, Senna P, Pozdnyakova O (2016) Automated nucleated RBC measurement using the Sysmex XE5000 hematology analyzer: frequency and clinical significance of the nucleated RBCs. Am J Clin Pathol 145:379-384

23. Ngoma AM, Saito S, Ohto H, Ikeda K, Yasuda H, Kawabata K et al (2011) CD34+ cell enumeration by flow cytometry: a comparison of systems and methodologies. Arch Pathol Lab Med 135(7):909914

24. Piedras-Ross J, León-Rodríguez E, Sánchez-Guerrero S, LópezKarpovitch X (2003) Comparison of single- and dual-platform approaches to enumerate CD34 Cells in bone marrow and mobilized peripheral blood stem cells. Arch Med Res 34:16-19

25. Kim H, Hur M, Choi SG, Moon HW, Yun YM, Hwang HS, Kwon HS, Sohn IS (2014) Performance evaluation of Sysmex XN hematology analyzer in umbilical cord blood: a comparison study with Sysmex XE-2100. Clin Chem Lab Med 52:1771-1779

26. Chapple PH, Wall D, Filshie R, Haylock D, Quinn M, Bretell M et al (2000) Comparison of three methods of CD34+ cell enumeration in peripheral blood: Dual-platform ISHAGE protocol versus single-platform, versus microvolume fluorimetry. Cytotherapy 2: 371-376

Publisher's note Springer Nature remains neutral with regard to jurisdictional claims in published maps and institutional affiliations. 